\title{
Observation of Critical Coupling in a Fiber Taper to a Silica-Microsphere Whispering-Gallery Mode System
}

\author{
Ming Cai, Oskar Painter, and Kerry J. Vahala* \\ Department of Applied Physics, California Institute of Technology, Pasadena, California 91125
}

(Received 17 December 1999)

\begin{abstract}
We present the observation of critical coupling in a high- $Q$ fused-silica microsphere whispering-gallery mode resonator coupled to a fiber taper. Extremely efficient and controlled power transfer to high- $Q$ $\left(\sim 10^{7}\right)$ resonators has been demonstrated. Off-resonance scattering loss was measured to be less than $0.3 \%$. On-resonance extinction in transmitted optical power through the fiber coupler was measured as high as $26 \mathrm{~dB}$ at the critical coupling point. This result opens up a range of new applications in fields as diverse as near-field sensing and quantum optics.
\end{abstract}

PACS numbers: 42.60.Da, 42.81.Qb

The condition of critical coupling is a fundamental property of waveguides coupled to resonators [1,2]. It refers to the condition in which internal resonator loss and waveguide coupling loss are equal for a matched resonator-waveguide system [3], at which point the resulting transmission at the output of the waveguide goes to zero on resonance. The use of a fiber taper waveguide [4] has several advantages in that it can be placed alongside a resonator allowing simple focusing and alignment of the input beam, as well as collection of the output beam. Most importantly, in the context of critical coupling, is that it very efficiently filters all other waveguide modes, save the fundamental, at both the input and the output. The mode filtering performed by the fiber taper before and after the coupling region results in a taper-resonator system which is for all intents and purposes a single-mode coupler and thus effectively an ideally matched coupler (nonidealities are lumped into the resonator round-trip loss).

Referring to Fig. 1, for ideal mode matching in the coupler [5], the taper-microsphere system can be described by a single coupling parameter $(t)$ and a resonator round-trip factor $(\alpha)$. A simple analysis [5] of the coupling results in the following expression for power transmission $T$ past the resonator:

$$
\begin{aligned}
1-T & =\frac{C}{1+f \sin ^{2}\left(\pi \frac{\nu}{\Delta \nu_{\mathrm{FSR}}}\right)}, \\
f & \equiv \frac{4 \alpha t}{(1-\alpha t)^{2}}, \\
C & \equiv 1-\left(\frac{t-\alpha}{1-\alpha t}\right)^{2},
\end{aligned}
$$

where $\Delta \nu_{\mathrm{FSR}}$ is the resonator free-spectral range [6], $f$ is the coefficient of finesse, and $C$ is the resonator coupling parameter. This result assumes that $\alpha$ has been suitably modified from the intrinsic resonator value to include losses associated with scattering into taper or radiation modes other than the fundamental taper mode. Observation and control of the critical condition are then dependent on several factors including the degree to which parasitic (i.e., not intentionally introduced) internal resonator loss as well as junction scattering "bias" the waveguide-resonator system relative to the critical point. Such parasitic losses can render the system permanently "undercoupled" (unless an amplifying medium is introduced). It can be seen that $C=1$ at the critical point (complete power transfer and dissipation of waveguide power), and thus near complete extinction is possible in a properly designed taper-microsphere system, limited only by the degree of filtering performed by the taper. This is in stark contrast to a prism coupler in which very little filtering is performed and most of the light scattered by the microsphere is collected. In this case extinctions of only $7 \mathrm{~dB}$ have been reported [3] due to the difficult task of mode matching.

Recently Gorodetsky and Ilchenko measured and varied the coupling in a prism-coupled microsphere by variation of the coupling gap [3]. In this paper we demonstrate control of resonator coupling in an optical-fibertaper to silica-microsphere whispering-gallery-resonator

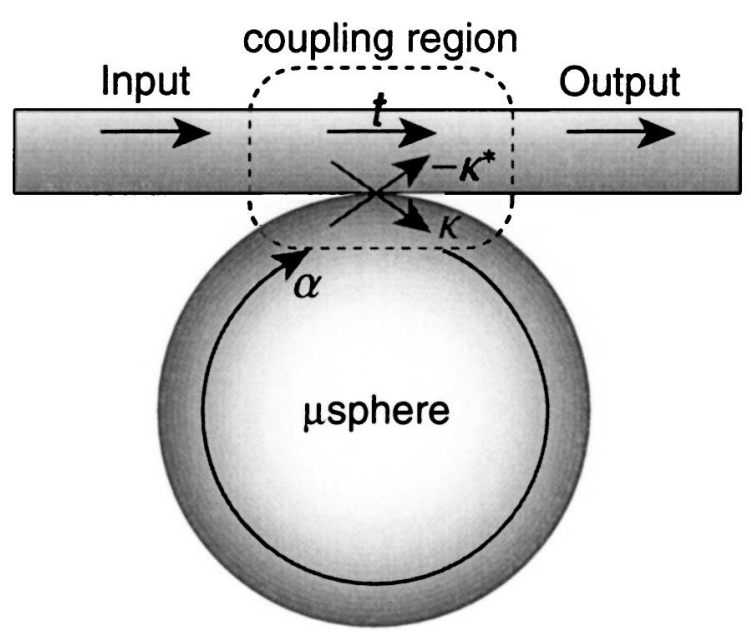

FIG. 1. Schematic of an ideal-matched fiber taper-microsphere system. $t$ is the transmission coefficient through the coupler and $\kappa=i \sqrt{1-t^{2}}$ is the taper-sphere mode coupling amplitude. $\alpha$ is the transmission coefficient for the field upon a round-trip in the resonator. 
system using controlled variation of the resonator loss. The optical extinction is measured to be as high as $26 \mathrm{~dB}$ in the vicinity of the critical point. Measurements are also performed of off-resonance insertion loss associated with the microsphere resonator being in contact with the fiber-taper waveguide. By proper selection of taper diameter negligible scattering loss (less than 0.3\%) is observed by the resonator, and strong overcoupling is demonstrated. These results are remarkable in that nearly lossless power transfer between fiber guides and high$Q$ optical resonators may provide an improved means to study quantum optical phenomena.

The spheres and fiber tapers studied were prepared from standard telecommunication fiber by a technique similar to that used in Refs. $[4,7,8]$. The tapers had diameters in the range of 3-5 microns. Microspheres with diameters ranging from 50-150 microns were fabricated by first preparing a sharp-tipped filament of fiber using a $\mathrm{CO}_{2}$ laser and then reheating the tip to achieve the desired sized sphere. The residual taper attached to the sphere then functioned as a holder to position the sphere relative to the fiber taper. The resonators were in physical contact with the taper during all measurements, unless specifically mentioned. A micrograph showing a typical microsphere resonator attached to a taper is shown in the inset in Fig. 2.

The whispering-gallery (WG) modes of silica microspheres have received considerable attention in the literature owing to their ultrahigh optical quality factors $[9,10]$. Ideal dielectric spheres have modes characterized by four indices $(n, l, m, p)$, where $(n, l, m)$ are radial and angular indices while $p$ designates either TE or TM polarization. In the ideal sphere each $(n, l, m)$ subspace is $2 l+1$ degenerate. Actual resonators exhibit slight eccentricity which splits the resonant frequencies into $2 l+1$ distinct values associated with the index $m$. This splitting is one of the most easily measured properties of the spheres. Figure 2 shows a typical transmission spectrum for the spheretaper system. This spectrum was obtained by measuring

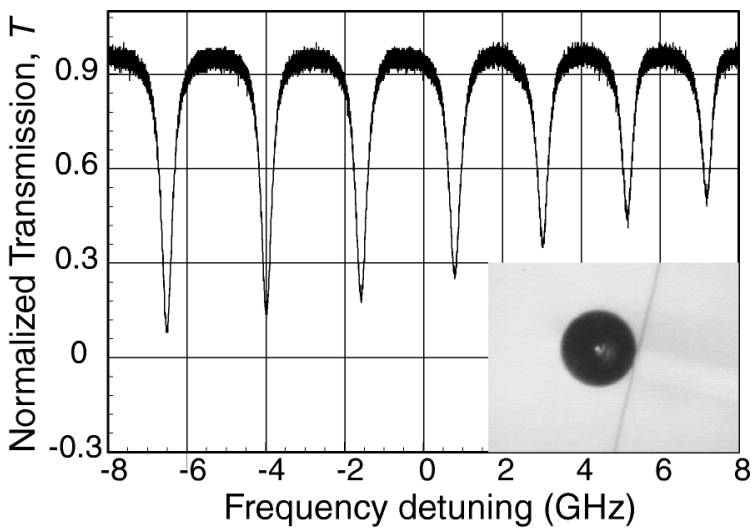

FIG. 2. Typical transmission spectrum for the microspheretaper system. The inset shows a magnified image of the fiber taper attached to the microsphere. the optical power transmission past the taper while scanning a single-frequency, tunable laser over a $15 \mathrm{GHz}$ span (around 1.55 microns). The transmission minima appearing in the spectrum of the inset in Fig. 2 are associated with different $m$ indices that have been split by resonator eccentricity.

To study critical coupling, a plastic probe having a smooth, round tip was prepared by slightly melting the tip of a multimode plastic optical fiber. The diameter of the probe tip was made comparable to the microsphere diameter so as to minimize probe-induced backscattering within the resonator. In addition, the plastic material was lossy in the 1.5 micron band so that power coupled from the resonator would be quickly absorbed within the probe. The probe was positioned near the microresonator using a piezoelectric motor with a $0.2 \mu \mathrm{m}$ resolution encoder for reading off the position of the probe. By approaching the probe to the surface of the microsphere, the total cavity loss of the resonant modes was increased due to the probe scattering and absorption.

Power transmission was measured for various WG modes in a $150 \mu \mathrm{m}$ diam silica microsphere as the probe position was varied in the vicinity of the microsphere. The fiber taper was fabricated with a diameter chosen to optimize phase matching (and thus coupling) to the fundamental $(m=l), n=1 \mathrm{WG}$ mode, and fine tuning of the matching was performed by moving the microsphere along the taper relative to the waist. By adjusting the taper position relative to the sphere equator and the input wave polarization we were able to select a low radial number, near fundamental $\mathrm{TE}_{n \approx 1, l, m \approx l}$ whispering-gallery mode for study.

Figure 3 shows the resonant transmission versus probe position. A clear minimum versus probe position is

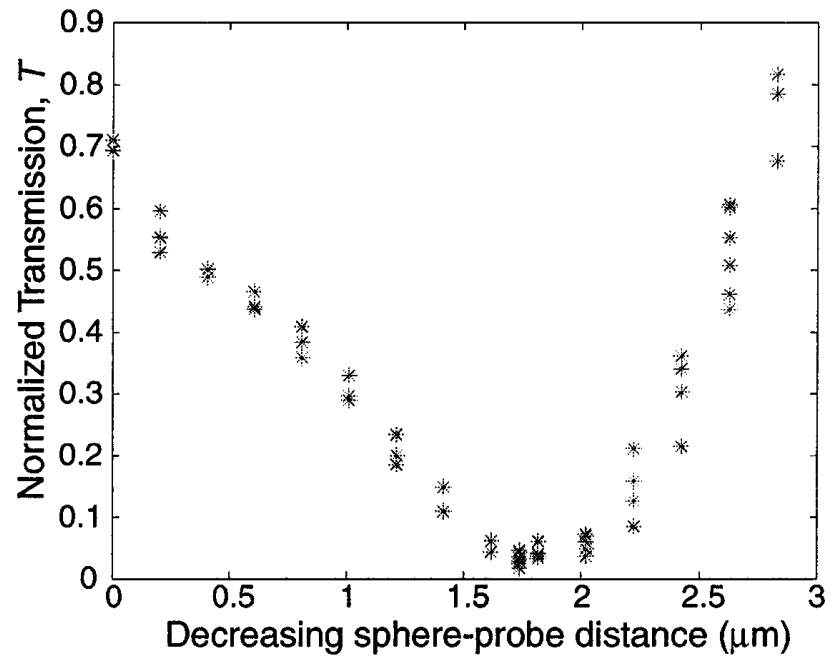

FIG. 3. Normalized transmission as a function of the probe position for a low radial number $(n \approx 1)$, near fundamental $(m \approx l)$ WG mode in a $150 \mu \mathrm{m}$ microsphere. The horizontal axis gives the change in the distance of the probe to the microsphere relative to an arbitrary position. 
apparent in the figure, corresponding to the critical point. Note that prior to the probe being brought into close proximity of the microsphere ( 0 point) the taper-microsphere system is biased well into the overcoupled regime $(T>70 \%)$. In fact overcoupled transmissions of greater than $95 \%$ were observed for optimum taper diameters, limited by our ability to accurately measure the transmission and linewidth due to overlap with other WG modes. The role of taper diameter here is believed to result from phase matched mode selection. This mechanism favors WG mode coupling to the fundamental taper mode as opposed to higher order taper modes. The ability to detect the critical point and to push the taper-microsphere system well into the overcoupled regime attests to the highly ideal nature of the taper-to-sphere junction.

Since the probe position is not linearly related to the mode loss (in addition there is variation in the motor step movement from step to step), a better way to plot the transmission data is shown in Fig. 4. In this plot, the on-resonance power transmission is shown plotted versus the linewidth of the resonant WG mode. Linewidth is a convenient measure of the mode loss and increases as the probe position is advanced towards the sphere. Transmission versus linewidth can also be easily calculated using the simple model of Fig. 1. For comparison, a calculation is provided in Fig. 4, where the transmission coefficient $(t)$ is fit to match the linewidth at the critical point. The critical coupling loss rate can be read directly off this kind of plot, giving it an even greater value to ascertain properties of the taper-sphere system. As an aside, note that the $Q$ values of the loaded sphere in this measurement are between $10^{6}-10^{7}$. The extremely sharp transition from overcoupling to the critical point and the correspondingly

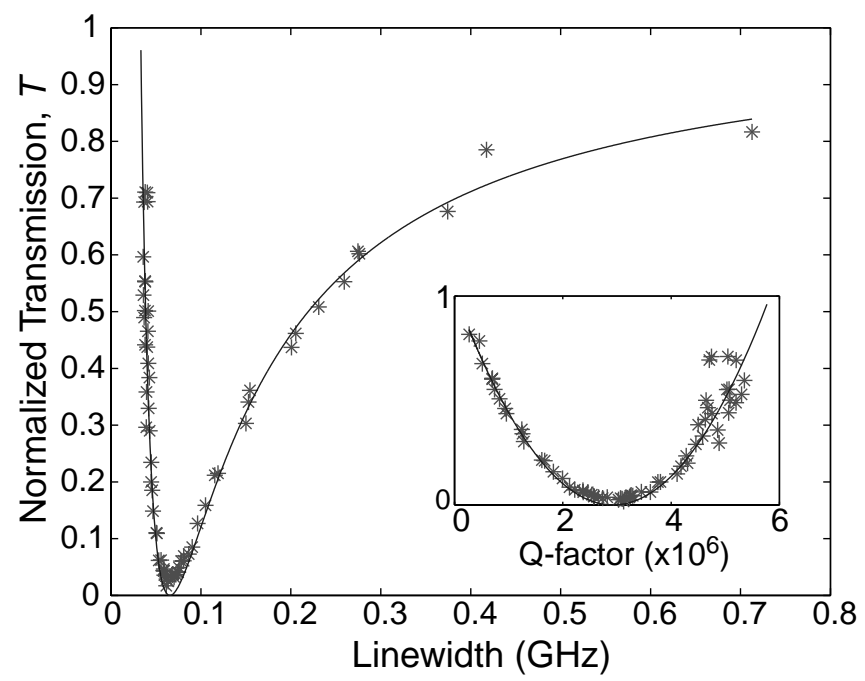

FIG. 4. Power transmission as a function of the linewidth (FWHM) of the mode for varying probe position (and resulting absorption). The solid line is calculated using the simple model of Fig. 1 with $t$ fixed. The inset shows the transmission as a function of the microsphere loaded $Q$. minute variation in resonator loss bode well for application of these structures as improved sensing devices [11].

Extinction in the vicinity of the critical point was difficult to assess owing to minute fluctuations in the probe's position affecting the power transmission. However, by covering the probe-taper-resonator system in a small box the stability improved sufficiently to enable more careful examination of the critical point region. The probe was set at a fixed distance from the sphere, and the taper-sphere gap was varied to adjust the coupling to the critical point. In this case the taper was very slightly detached from the sphere. A plot of the on-resonance transmission versus linewidth is shown in Fig. 5, along with a one-parameter fit in which the resonator round-trip amplitude $(\alpha)$ was chosen to match the linewidth at the critical point. Extinction as high as $26 \mathrm{~dB}$ in the transmitted power was measured, limited by the degree of control over the input wave polarization in the fiber.

Another important test of the junction is to measure the nonresonant insertion loss associated with the sphere being in contact with the taper. For this measurement, a trace of the transmission spectrum was stored using a digital oscilloscope both with and without the sphere in contact with the taper. The difference of the two traces was then computed to ascertain the insertion loss. By proper selection of the taper diameter this value was observed to be less than $0.3 \%$. The optimal taper diameters were in the range of 3 microns for the spheres studied here.

In conclusion, we have presented controlled critical coupling with $26 \mathrm{~dB}$ extinction in a high- $Q$ microsphere optical system. In addition, we observed strong overcoupling $(>95 \%)$ of the taper-sphere system as a result of phase matching of the fundamental taper mode to the

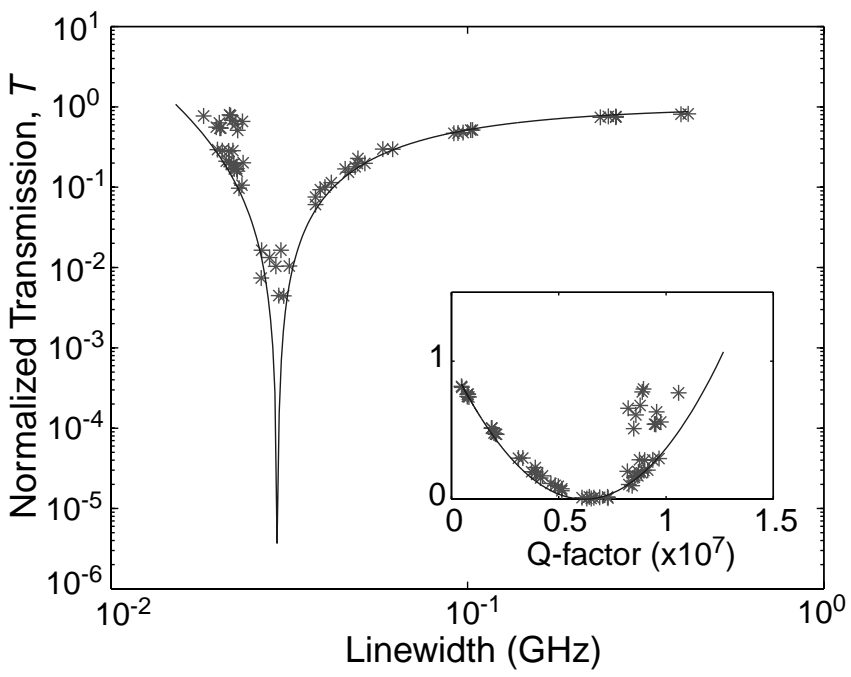

FIG. 5. Power transmission as a function of the linewidth (FWHM) of the mode for varying taper-sphere coupling gap. The solid line is calculated using the simple model of Fig. 1 with $\alpha$ fixed. The inset shows the transmission as a function of the microsphere loaded $Q$. 
microsphere WG mode. The results show that extremely efficient and controlled power transfer from a fiber waveguide taper to a high- $Q$ microsphere is possible.

This work was supported by DARPA and the Office of Naval Research. K. J. V. thanks A. Yariv for helpful discussions.

*Electronic address: vahala@its.caltech.edu; URL: http:// www.cco.caltech.edu/ aphhome/vahala.html

[1] R. Adler, L. J. Chu, and R. M. Fano, Electromagnetic Energy Transmission and Radiation (Wiley and Sons, New York, 1960).

[2] H. A. Haus, Waves and Fields in Optoelectronics (PrenticeHall, Englewood Cliffs, NJ, 1984).

[3] M. L. Gorodetsky and V.S. Ilchenko, J. Opt. Soc. Am. B 16, 147 (1999).
[4] J. C. Knight, G. Cheung, F. Jacques, and T. A. Birks, Opt. Lett. 22, 1129 (1997).

[5] Time reversal symmetry and energy conservation can be used to show that the corresponding four port coupling parameters on the resonator side are identical to the taper side and that $\kappa$ is purely imaginary.

[6] Here the free spectral range corresponds to the intermode spacing in $l$ of the microsphere whispering-gallery modes, where the effective cavity length is the circumference about the equator.

[7] M. Cai and K. Vahala, Opt. Lett. 25, 260 (2000).

[8] M. Cai, G. Hundziker, and K. Vahala, IEEE Photonics Technol. Lett. 11, 686 (1999).

[9] M. L. Gorodetsky, A. A. Savchenkov, and V. S. Ilchenko, Opt. Lett. 21, 453 (1996).

[10] D. W. Vernooy, V. S. Ilchenko, H. Mabuchi, E. W. Streed, and H. J. Kimble, Opt. Lett. 23, 247 (1998).

[11] P. Bridger, G. Hunziker, M. Cai, and K. Vahala, patent application. 